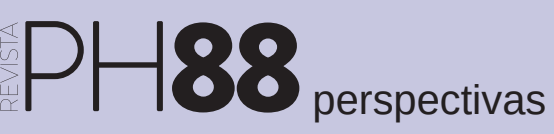

a debate La eficiencia energética y la edificación histórica | coordinan Mónica López Sánchez, Ana Yáñez Vega

\title{
Sostenibilidad y eficiencia energética de los edificios históricos
}

\author{
Elena Lucchi | investigador senior de la European Academy of Bolzano (EURAC), profesora en Politecnico di Milano \\ URL de la contribución <www.iaph.es/revistaph/index.php/revistaph/article/view/3665>
}

\section{INTRODUCCIÓN Y OBJETIVOS}

La cultura de la conservación y de la sostenibilidad del medio ambiente se caracteriza por raíces comunes que han dado lugar a una convergencia de objetivos, prácticas, métodos y herramientas. Ambas tienen como objeto de investigación el patrimonio cultural, un importante "recurso no renovable" que ha de ser valorizado a través de acciones compatibles con su valor histórico. Hay muchas razones para considerar la conservación y la recuperación de las viviendas históricas como una estrategia de desarrollo sostenible. De hecho, en muchos casos, los edificios históricos se encuentran en zonas densamente pobladas y equipadas con infraestructuras que responden ecológicamente a usos muy intensos. En general, los edificios son hechos con materiales locales y duraderos y se construyen después de un estudio detallado del medio ambiente, con el fin de explotar el potencial de los recursos naturales y renovables. Por eso, su recuperación tiene un alto porcentaje de embodied energy, que se puede definir como la cantidad de energía necesaria para extraer, recuperar y reutilizar materiales, objetos y edificios.

Más recientemente, el concepto de intervención sostenible se ha ampliado con la introducción de los criterios de eficiencia energética y confort. La primera puede ser considerada como un medio para proteger e incrementar el valor inmobiliario del patrimonio histórico. La renovación a un alto nivel energético puede tener un impacto negativo sobre la conservación y el valor estético del patrimonio, afectando a la estructura y a los espacios. Además, estas mejoras son generalmente costosas, en términos económicos y ecológicos. Un retrofit apropiado, por el contrario, tiene como objetivos la mejora energética, el bajo impacto, la valoración histórica y la regeneración económica y social de las zonas urbanas. Esta nueva visión ha hecho más compleja la intervención, ya que requiere el respeto y la apreciación de las cualidades estéticas, materiales y espaciales, así como la compatibilidad y la reversibilidad de los trabajos.

El conocimiento del edificio debe ser la base para la mejora energética. Los edificios históricos nacen de una estrecha relación con el entorno natural, basada en el estudio de características geométricas, variables climáticas (presión, temperatura y humedad relativa, estado del cielo, régimen del viento, precipitaciones, luminosidad...), parámetros geográficos (latitud, relación entre masa de aguas subterráneas y superficiales...), topográficos (altura, orientación, estructura del suelo, dirección de vientos predominantes) y biológicos (características de flora y fauna). El comportamiento energético y ambiental de un edificio histórico es muy diferente del moderno. En términos generales, incluso en climas muy diferentes, los edificios aprovecharon el aislamiento e inercia térmica de las paredes; fueron construidos con materiales transpirables e hicieron uso de ventilación e iluminación natural para asegurar el confort térmico, visual y olfativo. En la era industrial, el mayor rendimiento de los combustibles fósiles en relación con las fuentes renovables ha promovido la idea de energía inagotable, con bajo coste y libre de efectos negativos. El confort era garantizado por la presencia de aire acondicionado, aislamiento térmico e impermeabilización. Por eso, las mejoras posibles para un nuevo edificio pueden ser inadecuadas o peligrosas para uno antiguo.

Este artículo presenta los resultados del proyecto europeo 3ENCULT "Efficient energy for EU cultural heritage", cuyo objetivo es demostrar la viabilidad de la restauración de edificios históricos con estándares de alta eficiencia energética, garantizando el confort de las personas y la salvaguardia del patrimonio. El artículo tratará, en 
_a debate La eficiencia energética y la edificación histórica | coordinan Mónica López Sánchez, Ana Yáñez Vega

particular, del proceso completo de diagnóstico, diseño y mejora energética.

\section{MATERIALES Y MÉTODO}

La legislación sobre eficiencia energética ha crecido desde la última década, paralelamente a las iniciativas y recomendaciones a nivel europeo. El tema es complejo y no siempre se cuenta con el apoyo político para promover una legislación coherente y operativa.

En los últimos años, la Comisión Europea ha desarrollado un marco legislativo específico para reducir las emisiones de $\mathrm{CO}_{2}$ (DIRETTIVA 2002/91/CE; DIRETTIVA 2010/31/ UE), aumentar las fuentes renovables (DIRETTIVA 2009/28/UE) y mejorar el rendimiento energético de los edificios existentes (DIRETTIVA2012/27/UE) hasta el año 2020. En un futuro próximo, el marco legislativo implicará una aceleración importante de la recalificación energética de los edificios existentes. Las directivas europeas introducen sólo los principios generales relativos a las rehabilitaciones, dejando la aplicación de la legislación en manos de las autoridades de los Estados, de conformidad con la cultura nacional sobre la restauración arquitectónica. El patrimonio histórico, más que el contemporáneo, debe adaptarse a estos cambios físicos e intelectuales dentro de su entorno. En general, los "(...) bienes protegidos oficialmente por el interés histórico y arquitectónico" están excluidos de esta aplicación, cuando los requisitos de eficiencia energética pueden causar "(...) alteraciones inaceptables del carácter histórico o de su apariencia" (DIRETTIVA 2002/91/CE; DIRETTIVA 2010/31/UE). Los edificios históricos que no están protegidos son enteramente sometidos a estos dictados y deben cumplir con un rendimiento similar al nuevo edificio en términos de reducción de pérdidas por transmisión (un solo componente) y demanda de energía (todo el edificio). En la práctica, cuando se realiza una operación "importante" (demolición y reconstrucción, renovación, ampliación) se pide una mejora global sobre el edificio. Por el contrario, cuando se realizan pequeñas obras de reestructuración o ampliación es posible intervenir sólo con obras pasivas (bioclimática, ausencia de condensación en las paredes, inercia de la envolvente...).

Las políticas europeas y nacionales aun no responden claramente a la complejidad de los problemas de la eficiencia energética y confort ambiental en los edificios existentes, dejando muchas preguntas sin respuesta; por ejemplo, en relación con el significado de "alteración inaceptable", a la necesidad de diferenciar la performance de los edificios nuevos e históricos, a la posibilidad de graduar la intervención de acuerdo con la preservación de los valores históricos, a no considerar el ciclo de vida de los elementos técnicos...

Algunos países tienen también fondos económicos para ejecutar la Directiva EPBD (DIRETTIVA 2002/91/CE), acelerando su implantación. Esto ha causado una pérdida generalizada y acrítica de ventanas históricas (por ejemplo en Noruega, Finlandia, los Países Bajos, Gran Bretaña) (HOGELING, 2006; NYPAN; RONCHI, 2006). Basándose en la experiencia de Finlandia, donde la EPBD fue implementada de modo particularmente restrictivo, se creó un European Working Group "EU Directives and Cultural Heritage" que estudia los impactos que la legislación europea tiene sobre el patrimonio arquitectónico, con el intento de crear directrices comunes para los diferentes estados europeos (SPIEKMAN; VAN DIJK, 2011).

Por otro lado, algunos países (Reino Unido, Hungría, Dinamarca) han establecido controles y autorizaciones de organismos nacionales y locales, definiendo niveles de calidad, intervenciones y materiales posibles, con el fin de salvaguardar el patrimonio. A nivel nacional surgieron directrices (ENERGY, 2009; CONSERVATION, 2000) y protocolos (QUALITÀ, 2011; EFFICIENZA, 2013) que explican las técnicas para aumentar la eficiencia energética, conservando los valores y sin comprometer la estructura histórica. En Italia se definió un protocolo voluntario (GBC Historic Building ${ }^{\mathrm{TM}}$ ) para certificar la sostenibilidad de las intervenciones (PROTOCOLLO, 2014), que ha servido como base internacional. A nivel europeo, sin embargo, los programas financieros se centran en la ciudad (JESSICA - Joint European Support for Sustainable 
a debate La eficiencia energética y la edificación histórica | coordinan Mónica López Sánchez, Ana Yáñez Vega

Investment in CityAreas; BEl - Smart grid), y la recuperación de la eficiencia energética y la cohesión social (fondos estructurales).

\section{METODOLOGÍA OPERACIONAL}

El diagnóstico de las características históricas, texturales, artísticas y energéticas es la base para definir las mejoras más apropiadas para un edificio histórico. Una buena práctica consiste en la integración de las técnicas de restauración, auditorías energéticas, evaluación de prestaciones, análisis del confort, con el fin de conseguir una visión global del estado de conservación. Se trata de un "proceso sistemático" de conocimiento de la construcción original: sus modificaciones, sus condiciones de uso, su estado de conservación, sus cualidades, sus valores materiales e inmateriales, así como las carencias y las oportunidades para su reconversión (LUCCHI, 2012). El método de valoración comienza desde el análisis de la literatura (THUMANN; JOUNGER, 2008; DALL'O', 2011; CEI EN 16247-1) y consiste en las siguientes fases:

$>$ análisis de los documentos históricos;

$>$ análisis funcional del edificio;

> auditorías energéticas y simulación termodinámica;

> definición de las acciones de reconversión más adecuadas;

> evaluación de la compatibilidad estructural, constructiva, física y económica de cada acción;

> evaluación de la viabilidad tecnológica y económica de las acciones seleccionadas;

> planificación estratégica de las actividades;

> identificación y transmisión de los beneficios de conservación, exposición y gestión a los usuarios.

\section{Análisis documental}

El análisis documental de la evolución histórica del edificio permite comprender la historia que tuvo el edificio a través del tiempo, justificando la presencia de determinadas tecnologías, materiales, métodos de instalación y daños. Se compone de las siguientes etapas:

> encuesta geométrica;
> análisis histórico y documental;

> estado de conservación de los materiales;

> análisis de las patologías de degradación.

Las informaciones geométricas se recogen fácilmente, incluso en ausencia de dibujos del proyecto, una situación especialmente común en los edificios antiguos. Por eso es necesario crear una encuesta geométrica del tamaño del edificio, estudiando las relaciones entre diferentes espacios, superficies dispersantes (paredes, ventanas, puertas, suelos), alturas y volúmenes interiores... Este paso debe acompañarse de una investigación histórica que documente la evolución de la estructura con estudios documentales y pruebas sobre la estratigrafía de las paredes. Este tipo de análisis puede justificar la presencia de determinadas tecnologías, materiales, métodos de edificación, falta de homogeneidad y daños. El registro histórico, generalmente disponible para los edificios monumentales, difícilmente lo está para el patrimonio extendido para el cual no hay documentos y testimonios históricos. El análisis de los materiales ofrece información adicional sobre el tipo, el estado de conservación y la compatibilidad química y física entre el edificio y los nuevos materiales y componentes. El análisis del estado de conservación permite entender la existencia de deterioro, con el fin de restablecer el edificio.

\section{Análisis funcional}

El análisis funcional es particularmente útil para definir el uso futuro, en relación con las posibilidades y límites reales del edificio en su estado actual. La inclusión de nuevas características puede ser crítica para determinar si existe congruencia entre las necesidades y las posibilidades reales de la construcción, sin cambiar los valores y significados que ha tenido a lo largo de la historia.

\section{Diagnosis energética}

La auditoría energética es “(...) un procedimiento sistemático para conocer adecuadamente el perfil de consumo de un edificio (...) para identificar y cuantificar las posibilidades de ahorro energético partiendo de un análi- 


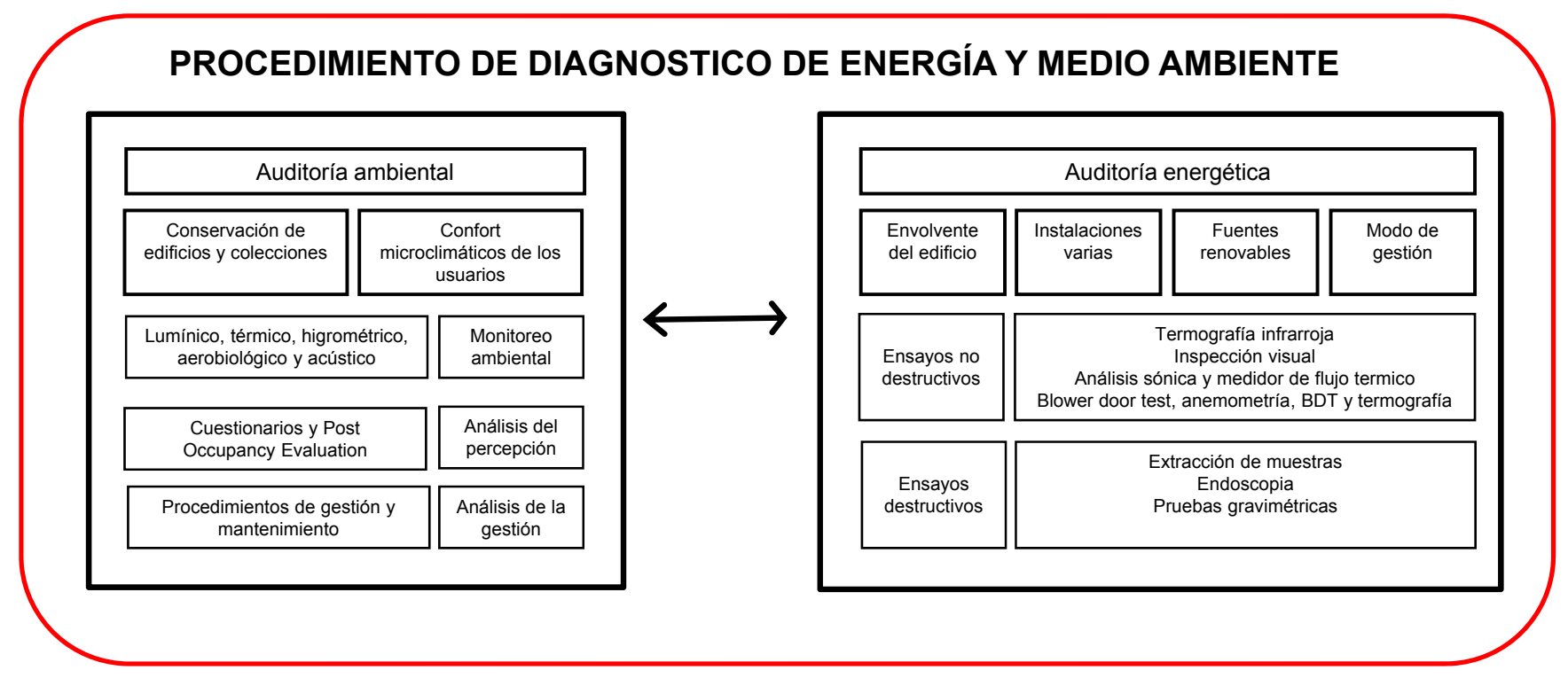

Procedimiento integrado de auditoría energética y ambiental basado en el uso de diferentes técnicas diagnósticas instrumentales para conocer detalladamente las propiedades energéticas del sistema edificio-instalación | todos los esquemas de este artículo son de elaboración propia, salvo que se indique lo contrario

sis costes-beneficios (ACB) que informe sobre los resultados" (DIRETTIVA 2012/27/UE). En un edificio existente es una herramienta muy importante para investigar las características y el nivel de eficiencia energética, así como para identificar las intervenciones más apropiadas. La literatura de referencia ha definido un proceso de auditorías energéticas de edificios existentes que se utiliza a nivel internacional (THUMANN; JOUNGER, 2008; DALL'O', 2011). No hay un procedimiento específico para los edificios históricos, aunque algunas primeras investigaciones se están moviendo en esta dirección (LUCCHI, 2012; LUCCHI; PRACCHI, 2013). El procedimiento consiste en cuatro etapas:

1. Recopilación de una amplia gama de información relativa a los aspectos geométricos (dimensiones, volúmenes), de construcción (características prestacionales de la envolvente opaca y transparente), instalación (prestaciones de sistemas de calefacción, aire acondicionado, ventilación, iluminación y sistema eléctrico) y de gestión del inmueble (horarios y días de funcionamiento de las instalaciones, temperaturas operativas, consumos de energía).
2. Simulación del comportamiento energético del edificio y evaluación de los flujos reales de energía.

3. Identificación de las mejoras más adecuadas para reducir el consumo de energía y optimizar el confort ambiental.

4. Evaluación técnica y económica de la viabilidad de las mejoras propuestas.

Para obtener información más detallada sobre el funcionamiento energético del edificio y de ciertas condiciones, es necesario el uso de alguna técnica diagnóstica adicional. Las pruebas instrumentales se dividen en dos tipos principales: ensayos no destructivos y destructivos.

Los ensayos no destructivos son el conjunto de exámenes, pruebas y encuestas realizadas con técnicas instrumentales que no alteran el material y que no requieren la extracción de muestras, con el fin de identificar defectos estructurales, mal funcionamiento y otros problemas. Las técnicas que se utilizan son: examen visual, termografía infrarroja, análisis sónico y gas penetrante. Para obtener resultados cuantitativos sobre la performance energética de la evolvente, los perfiles de uso y las con- 


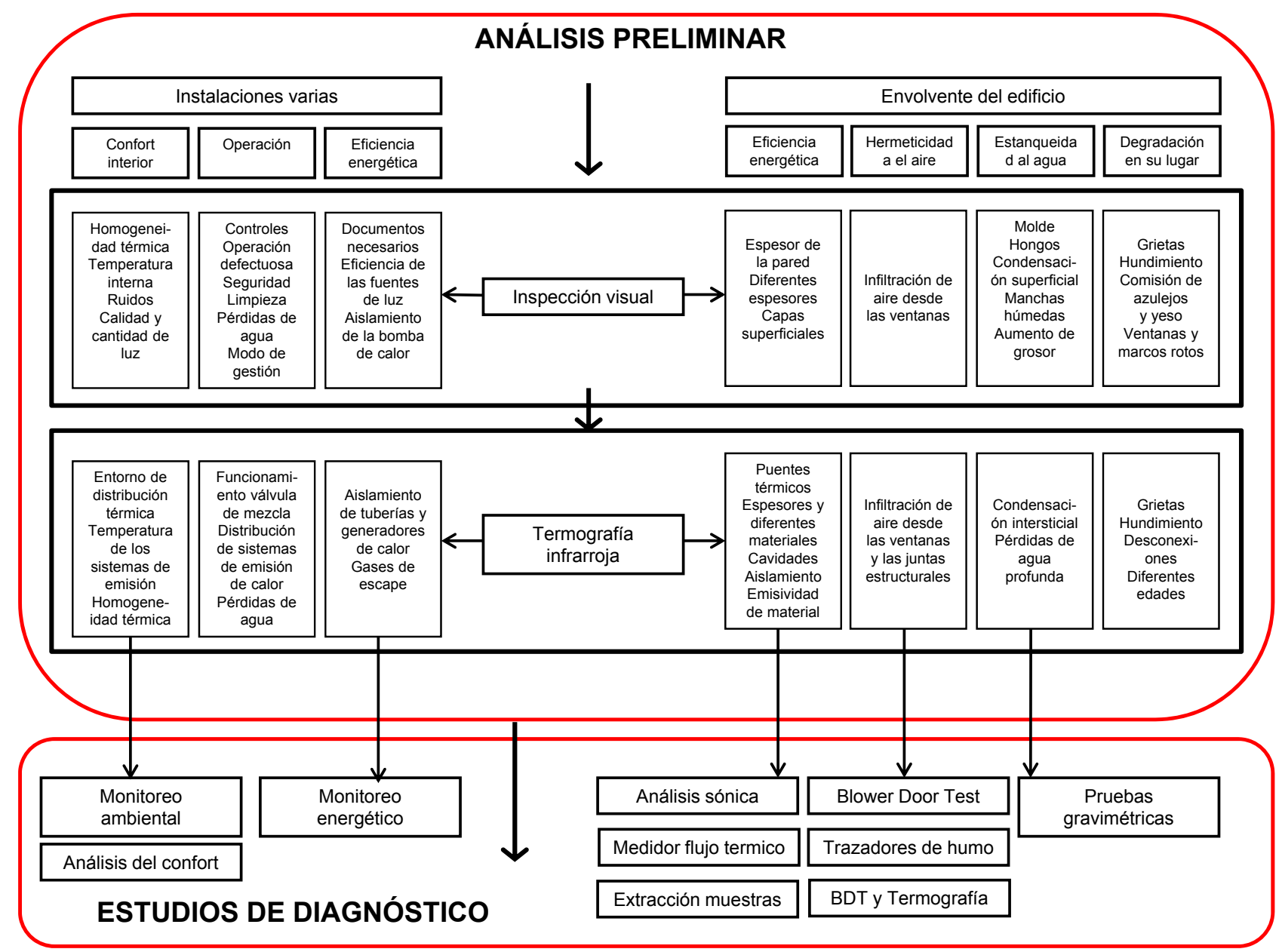

Proceso de estudio preliminar realizado integrando el examen visual y la termografía infrarroja

diciones micro-climáticas internas, estas pruebas son apoyadas por el Blower Door Test, el medidor térmico de flujo y el monitoreo ambiental y energético (LUCCHI, 2012). La termografía infrarroja mapea la temperatura superficial aparente de un objeto, identificando las anomalías térmicas superficiales que representan diferentes períodos históricos, tipos estructurales, materiales constructivos, puentes térmicos, obstrucciones de aberturas o elementos estructurales, presencia de chimeneas, continuidad del aislamiento térmico, infiltración de aire o agua, funcionamiento de instalaciones mecánicas, eléctricas, solar fotovoltaica y térmica (IS0 6781). El examen de la estratigrafía de los muros puede ser apoyado por la prueba sónica que caracteriza y describe la calidad de la mampostería, a través de la propagación de las ondas elásticas en las paredes. Esta prueba permite conocer la tipología de construcción, la composición física, la presencia de materiales y aislamiento y la ejecución de obras de restauración aunque sea una pared muy espesa. La transmitancia y la conductividad térmica se pueden calcular con el medidor del flujo de calor, que mide la resistencia, la conductividad, la transmitancia térmica y los efectos de la humedad en el rendimiento térmico de la evolvente (ISO 9869). Los aspectos de la ventilación natural y artificial se pueden evaluar con el Blower Door Test, que estima las infiltraciones y 

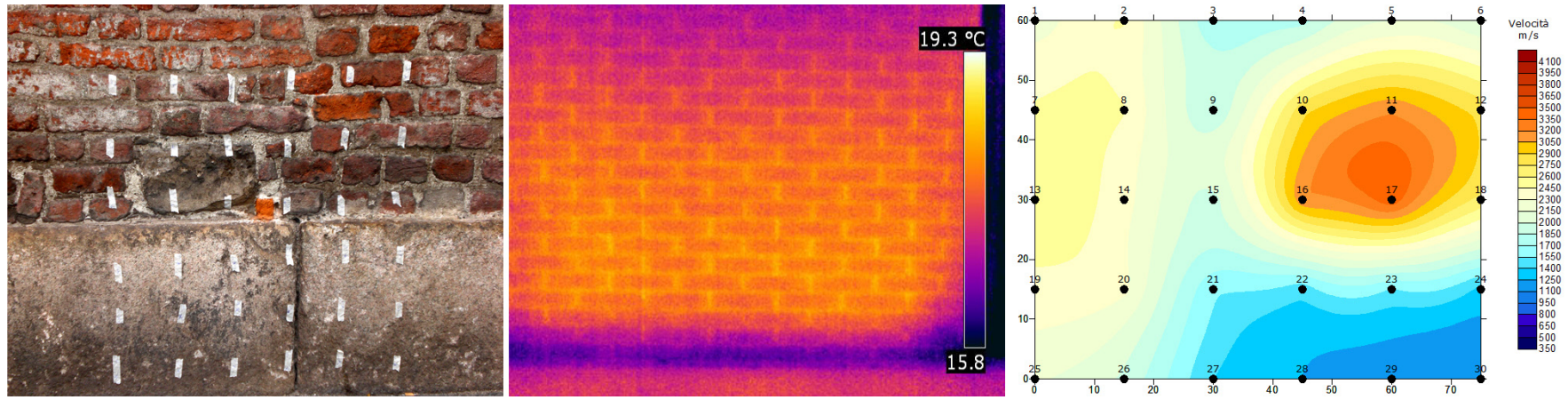

La auditoría energética se realizó en una pared antigua de gran espesor e inercia térmica. El diagnóstico fue apoyado por una investigación de los aspectos tecnológicos y morfológicos del edificio, con el fin de descubrir las técnicas de construcción tradicionales. La termografía infrarroja mostraba sólo la presencia de los ladrillos comunes. El análisis sónico ha demostrado la presencia de anomalías mecánicas, confirmando la estructura compuesta de la pared con ladrillos, piedras y mezcla de material
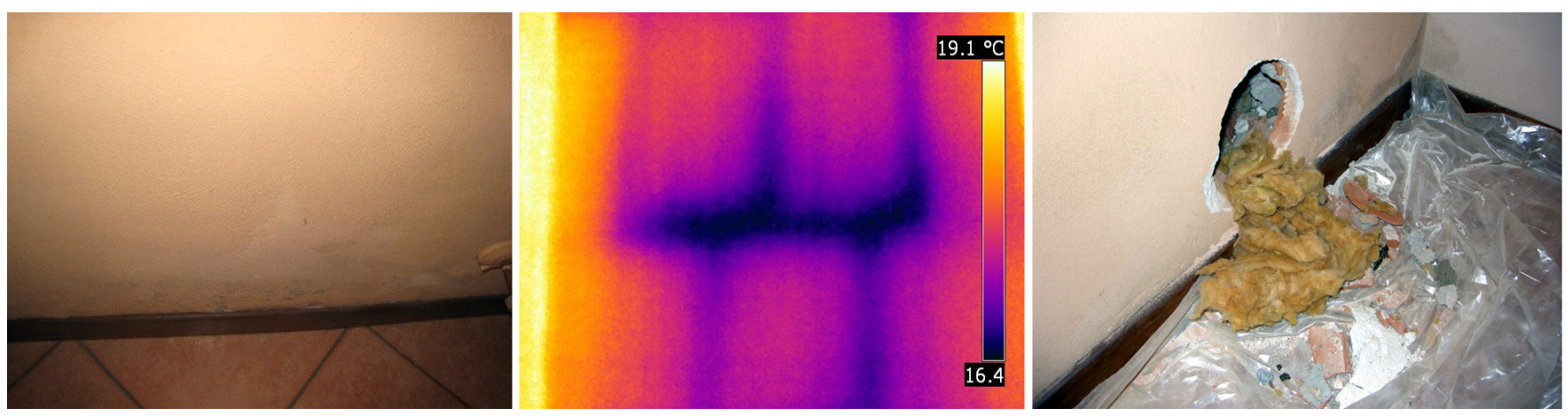

La auditoría energética se realizó en un edificio con problemas de anomalías térmicas, ineficiencia, humedad, moho, absorción de agua y grietas en la envolvente. El problema era tan inusual que el examen visual y la termografía infrarroja pueden crear un malentendido. Anomalías térmicas pueden interpretarse como una falta de aislamiento. Sólo el uso de extracción de muestras mostró la presencia de humedad y agua de percolación en el aislamiento térmico
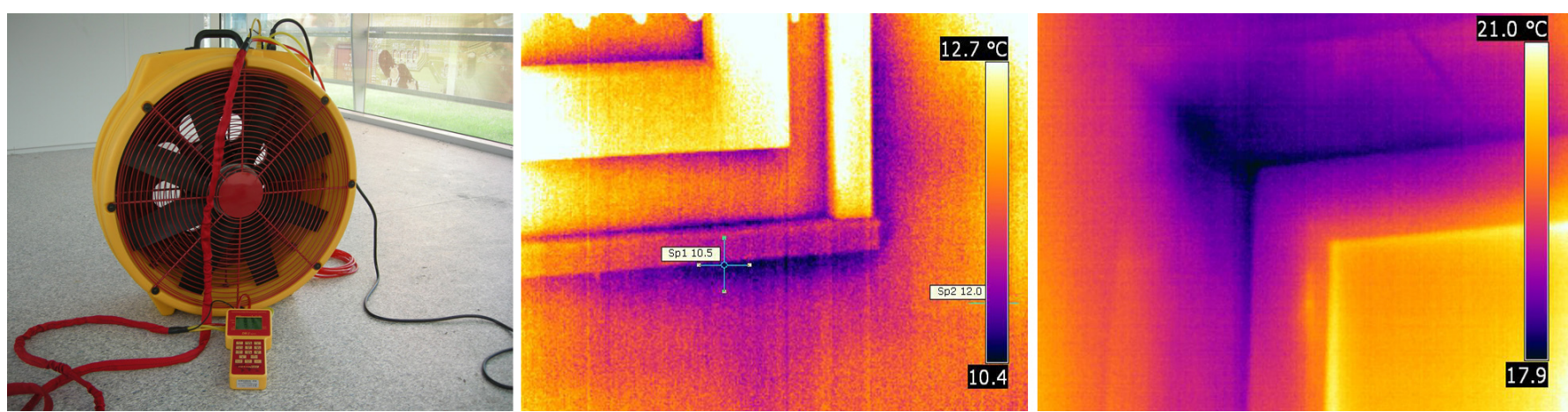

Instrumentación para el Blower Door Test y dos ejemplos de infiltraciones del aire desde una puerta y una ventana 
el intercambio del aire (ISO 9972). Se pueden utilizar, junto con la termografía infrarroja, los gases trazadores y la anemometría. En el primer caso, muestra la infiltración de aire y la pérdida de calor por ventilación (por ejemplo: tomas de corriente, iluminación, techos y juntas estructurales, puertas, ventanas). Los gases trazadores destacan la presencia de grietas o elementos con mala estanqueidad (puertas, ventanas, juntas estructurales, falsos techos, suelos elevados), desde donde se puede ver el humo. Por último, la anemometría se utiliza para medir la velocidad del aire en puntos definidos.

La evaluación ambiental considera exigencias opuestas para cuidar y valorizar el patrimonio cultural y garantizar el confort de los usuarios. La información relativa a la gestión, estado de conservación y funcionamiento de instalaciones puede obtenerse mediante una inspección visual. Al mismo tiempo, el monitoreo ambiental proporciona información sobre el uso real de los usuarios, ya que permite controlar el nivel de iluminación, luminancia, radiación UV e IR, temperatura, humedad relativa, sonido, calidad del aire y cantidad de $\mathrm{CO}_{2}$ en su interior, durante el año entero. Finalmente, el conocimiento de los horarios de apertura, operaciones y número de personas permite verificar la presencia de daños debido a un gran flujo de usuarios $y$, a veces, la incompatibilidad entre las políticas de uso y la protección del edificio histórico. La falta de integración entre las diferentes técnicas puede producir una interpretación incorrecta de los problemas específicos.

La información debe entonces ser reprocesada a través del uso de herramientas de simulación para comprender el rendimiento y el consumo de energía. La complejidad de estos temas requiere el uso de sistemas de simulación informática, que modelan el comportamiento del edificio y luego definir los trabajos más apropiados para equilibrar las necesidades de conservación, confort, eficiencia energética y sostenibilidad ambiental. Los sistemas actualmente en uso son:

> Cálculo en régimen casi estacionario que implica simplificaciones en la introducción de datos de intercambio térmico que afectan a la construcción.
> Cálculo en régimen dinámico que considera intervalos breves de tiempo (por ejemplo, una hora), a fin de considerar el calor acumulado y liberado por la masa del edificio.

Los primeros se utilizan para certificar el comportamiento y la clase de eficiencia energética de los edificios. Su aplicación a construcciones históricas presenta algunos problemas relativos a la simplificación de las características geométricas, climáticas y termofísicas y a la exclusión de toda la información inherente a la gestión del edificio. Las limitaciones más graves se refieren a la presencia de bases de datos referidas a materiales y técnicas modernas, a la imposibilidad de considerar los beneficios de la inercia térmica de paredes masivas, a la dificultad de simular edificios sin calentamiento o aire acondicionado y a la presencia de procedimientos de cálculo simplificado. Por lo tanto, estos sistemas son demasiado aproximados si se aplican a edificios. En el Reino Unido se estableció una metodología simplificada y un software para la certificación de edificios históricos, que estima el rendimiento energético basado en el periodo histórico y las técnicas de la construcción (RdSAP). Hay también guidelines que explican la evaluación de los resultados y posibles intervenciones (English Heritage). En Alemania, el Passivhaus Institut ha desarrollado un procedimiento (EnerPHit) para edificios existentes, actualizando también el software de simulación con datos y escenarios apropiados para el retrofit del patrimonio.

Los softwares que operan en régimen dinámico analizan simultáneamente flujos térmicos, eléctricos, lumínicos y de ventilación, el uso y todos los parámetros que influyen en el comportamiento térmico del edificio. Son herramientas abiertas que, para modelar correctamente, requieren de una precisión extrema en la introducción de datos. El principal problema se refiere sobre todo a la recuperación de datos energéticos precisos de la envolvente y de las instalaciones. Los estándares normativos internacionales y europeos, los programas de construcción y las referencias de la literatura son demasiado generales e inadecuadas para los edificios histó- 
a debate La eficiencia energética y la edificación histórica | coordinan Mónica López Sánchez, Ana Yáñez Vega

ricos. Además, los datos necesarios en la modelación dinámica se pueden recuperar sólo de manera parcial con las pruebas de diagnóstico in situ o en laboratorio. La información sobre la envolvente edilicia no se puede encontrar tan fácilmente con los análisis instrumentales no destructivos que, si por un lado son más compatibles con la construcción histórica, por el otro procuran preferentemente informaciones de tipo cualitativo, muy diferente de lo que necesita el software de tipo dinámico. De forma análoga, esta información se puede encontrar solo parcialmente con las pruebas de diagnóstico de tipo destructivo que, sin embargo, no siempre son compatibles con el bien histórico y, en cualquier caso, son muy largas y costosas. En ambos tipos de pruebas también es difícil recoger información sobre el funcionamiento de las instalaciones antiguas, donde hay pocos análisis históricos o ninguna referencia técnica. Por otra parte, es muy complicado modelar situaciones "anormales", como las chimeneas antiguas o la ventilación natural de una ventana mal sellada o siempre abierta. De la misma forma, las dimensiones relevantes y la presencia de puertas y ventanas mal cerradas no permiten

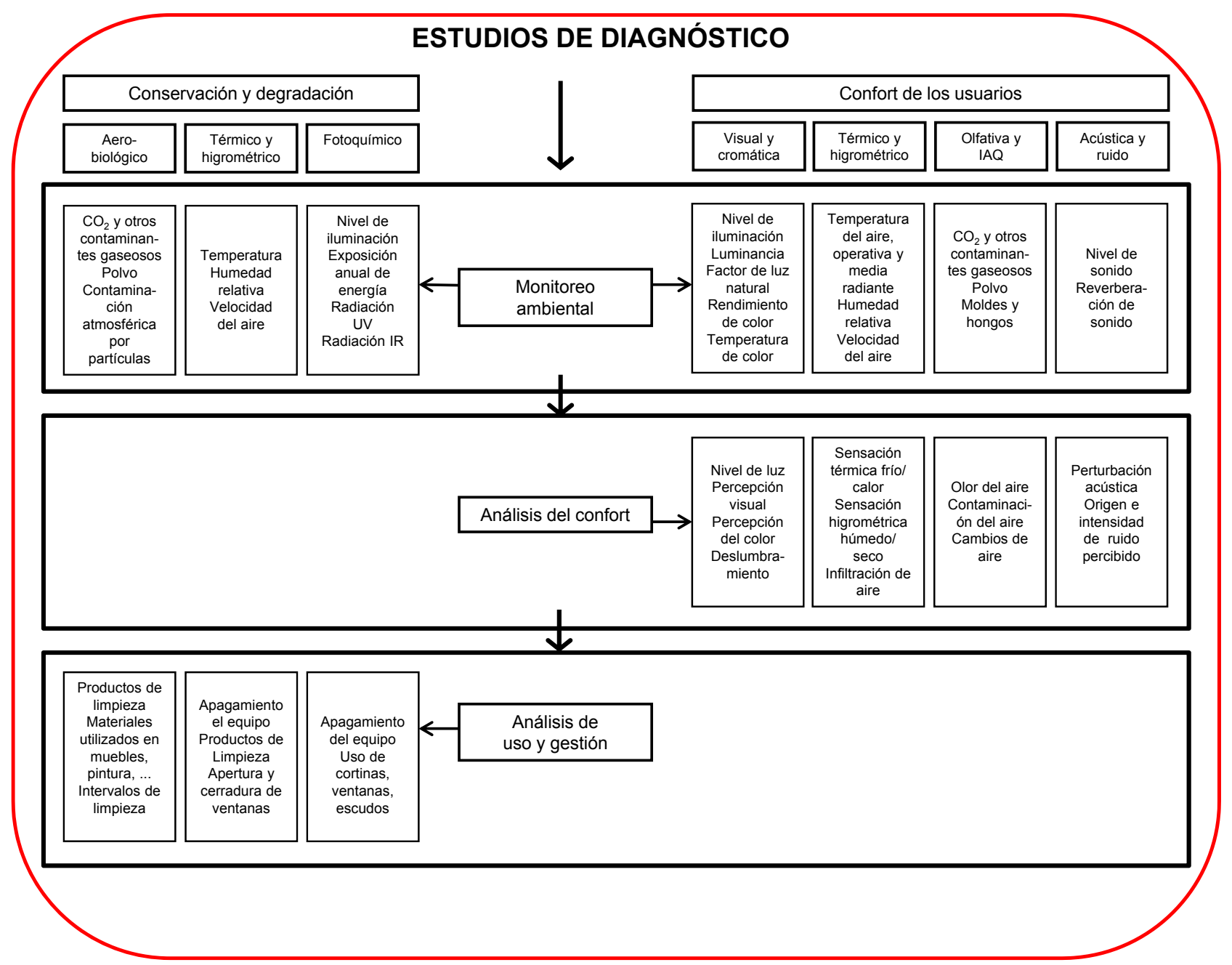

Proceso de estudio avanzado realizado integrando diferente tipos de monitoreo ambiental 
obtener resultados fiables sobre la infiltración del aire, incluso utilizando Blower Door Test, humos trazadores o anemometría. Por último, la presencia de alteraciones o degradés, cuyo comportamiento energético puede ser difícilmente simulado, puede cambiar de manera significativa los resultados de la modelación.

Para tal fin, es crucial calibrar el modelo con los datos obtenidos con el diagnóstico instrumental y los algoritmos apropiados. Esta información debe utilizarse como dato de entrada para construir el modelo (por ejemplo, los datos prestacionales de la envolvente que derivan de los análisis sónicos, del flujo de calor e infrarrojos y del con- tenido de humedad de las pruebas gravimétricas, entre otros) y de salida para verificar su funcionamiento (por ejemplo, la temperatura, la humedad relativa y el consumo energético modelados deben descostrarse poco respectivamente desde el monitoreo in-situ y las facturas de energía). El modelado dinámico se puede utilizar como una herramienta para comparar dos escenarios, con el fin de definir los beneficios de cada tecnología en relación con los requisitos globales de energía del edificio.

$\mathrm{Si}$, por un lado, las técnicas y procedimientos diagnósticos han alcanzado niveles muy avanzados también aplicados al patrimonio cultural, por el otro la simulación

\begin{tabular}{|c|c|c|c|}
\hline Acción & Compatibilidad & Integración & Reversibilidad \\
\hline \multicolumn{4}{|l|}{ Muros } \\
\hline Aplicar ETICS & - & - & - \\
\hline Insertar doble fachada & - & - & - \\
\hline Aislar con yeso térmico el exterior & - & $\square$ & - \\
\hline Aislar con yeso térmico el interior & - & - & - \\
\hline \multicolumn{4}{|l|}{ Sótano } \\
\hline Aplicar el aislamiento inferior & - & - & - \\
\hline Reemplazar el sótano & - & - & - \\
\hline \multicolumn{4}{|l|}{ Techos } \\
\hline Reemplazar con techo aislante o verde & - & घ & - \\
\hline Aplicar aislamiento exterior & - & घ & - \\
\hline Aplicar aislamiento interior & - & - & - \\
\hline Instalar aislamiento en el techo & - & - & - \\
\hline \multicolumn{4}{|l|}{ Ventanas y puertas } \\
\hline Reemplazar con sistemas de alta eficiencia & - & - & - \\
\hline Instalar cristales de baja emisividad en los marcos existentes & - & - & - \\
\hline Instalar las contraventanas & - & $\square$ & - \\
\hline Instalar películas sobre los cristales & घ & घ & घ \\
\hline Instalar burletes en las ventanas & - & $\square$ & $\square$ \\
\hline Reparar los sistemas existentes & घ & a & घ \\
\hline
\end{tabular}

Nomenclatura: $\mathbf{m}=$ Sí; - = No; $\mathbf{\square}=$ Se requiere un proyecto específico 
dinámica requiere más estudio, con el desarrollo de un database específico para el patrimonio y modelos que simulan la inercia térmica de paredes y la ventilación típica de un edificio histórico.

\section{Retrofit energético}

La lógica de la intervención tiene que reducir al mínimo las pérdidas de transmisión a través de las paredes, el rendimiento de las ventanas para transmisión y ventilación, los consumos de calefacción y sistemas eléctricos $y$, finalmente, la inclusión de fuentes renovables. A continuación vamos a discutir algunas de las intervenciones
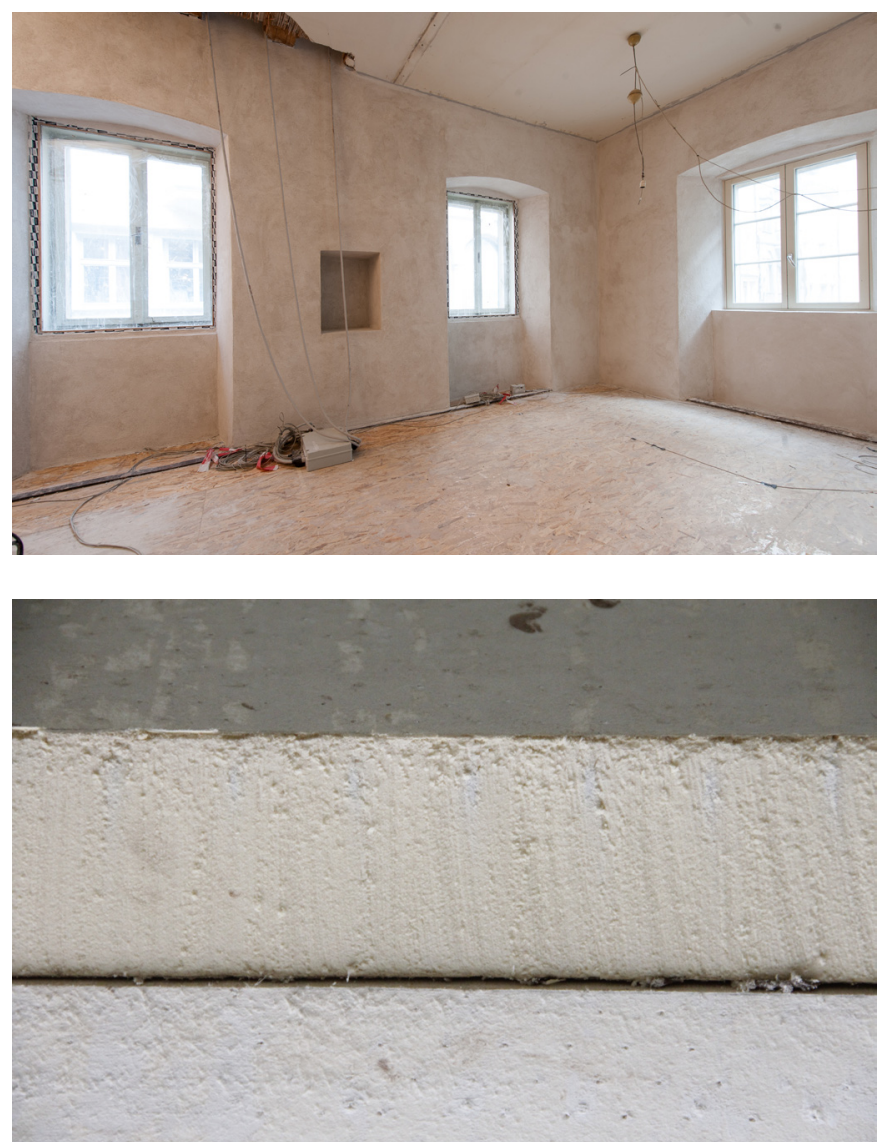

Aislamiento interior con materiales a capilaridad activa que difunden y trasportan la humedad $(\lambda=0,031 \mathrm{~W} / \mathrm{mK})$ | fotos Florian Berger/EURAC pasivas para ser implementadas en la envolvente del edificio (ver tabla de página anterior).

\section{Paredes}

Una primera forma de eficiencia energética se refiere a la aplicación de un aislamiento externo o interno de las paredes. El primero es difícil de aplicar, ya que cambia la imagen, la estética y la consistencia del material, resulta irreversible y difícil de conciliar con las características químicas y físicas de la pared original. En general, sin dejar de ser poco factible, reduce el consumo de energía, elimina los puentes térmicos de materia, explota plenamente las características de inercia térmica de las

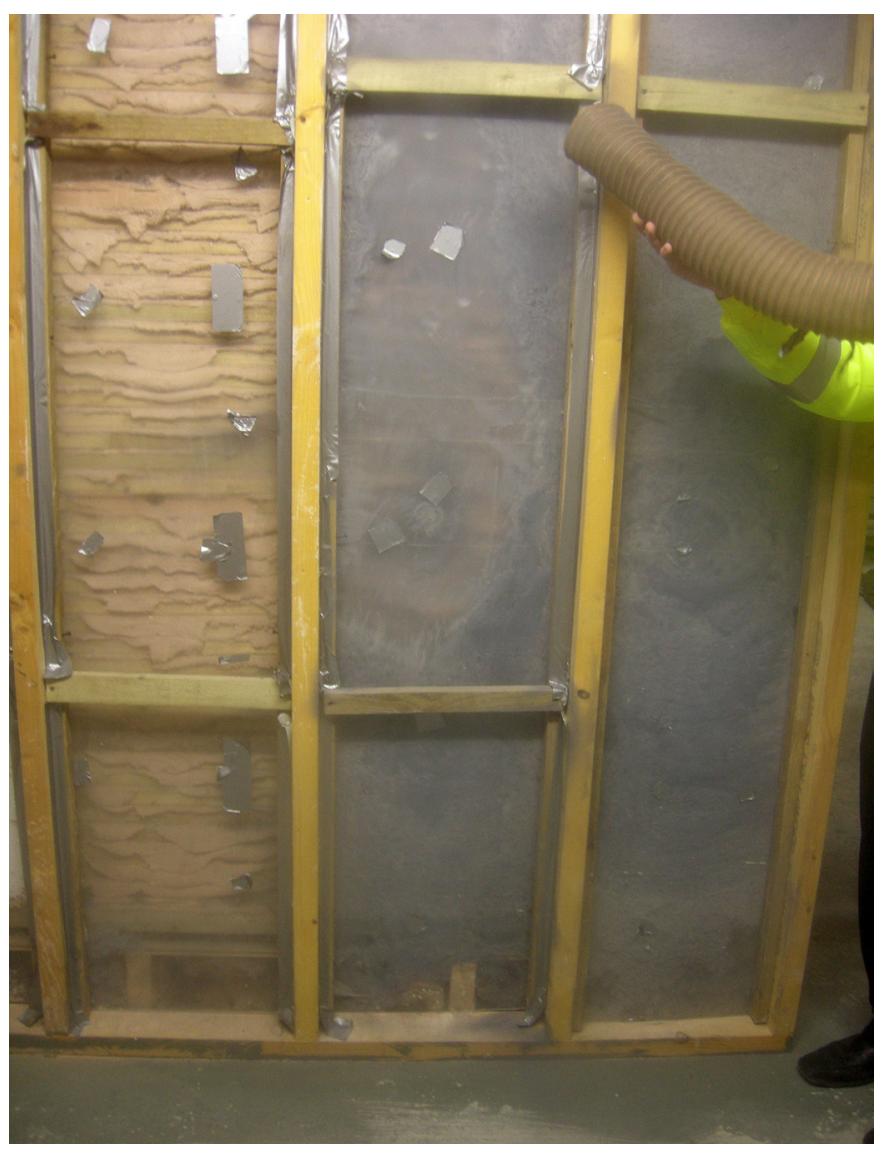

Aerogel hecho de fibra de poliéster, a la que se aferran partículas de aerogel, para combinar rendimiento térmico, permeabilidad al vapor, higroscopicidad y resistencia mecánica $\left(\lambda=0.255 \mathrm{~W} / \mathrm{mK}\right.$ con densidad de $\left.70 \mathrm{~kg} / \mathrm{m}^{3}\right)$ | foto Elena Lucchi 
estructuras masivas y reduce el riesgo de condensación. El aislamiento interior, siendo más fácil de implementar, tiene una eficiencia inferior, ya que no elimina los puentes térmicos y no aprovecha al máximo la inercia de los muros. En ambos casos, es preferible utilizar materiales de espesor reducido que garantizan elevados aislamientos térmicos y buenas transmisiones de vapor, como materiales con capilaridad activas, aerogel, morteros y pinturas nano-poliméricas. Materiales con alta capacidad de calor, tales como materiales de cambio de fase y madera, son adecuados para los edificios con baja inercia, como paredes de madera y de ladrillo con espesor reducido, típicos de la arquitectura moderna. En todos los casos es necesario verificar el impacto estético del sistema, la resistencia mecánica y estructural de la pared existente, la compatibilidad termohigrométrica y la reversibilidad del nuevo material.

\section{Suelos y cubiertas}

El aislamiento de plantillas, bases y cubiertas implica la inserción del material en la cavidad o la aplicación de un falso techo aislado. En el primer caso, el material que se utilizó para endurecer las plantillas se puede sustituir con aislamiento interno (vermiculita, perlita, arcilla expandida), que garantiza la ligereza y la estática, y que aumenta el poder aislante del sonido. En el segundo, cuando no hay decoraciones especiales, se puede colocar un aislamiento del techo o recuperar la estructura histórica con aislante (por ejemplo en bambú). La reparación de la cubierta debe ser vista como una oportunidad cuando hay una seria degradación, para mejorar su rendimiento con aislamiento o impermeabilización.

La reconstrucción de la cubierta podría ser la ocasión para incluir fuentes de energía renovables. En este caso, es necesario promover la integración arquitectónica, mecánica y tecnológica de la energía solar fotovoltaica, térmica y eólica. Algunos países europeos (Alemania, Austria, Italia, Suiza) han hecho unas directrices comunes, que explican los principios para la máxima integración con el paisaje y el edificio. En general, los principios son: plenitud, respeto de las líneas, forma regular y ordenada, reducido impacto visual, respeto de los colo-
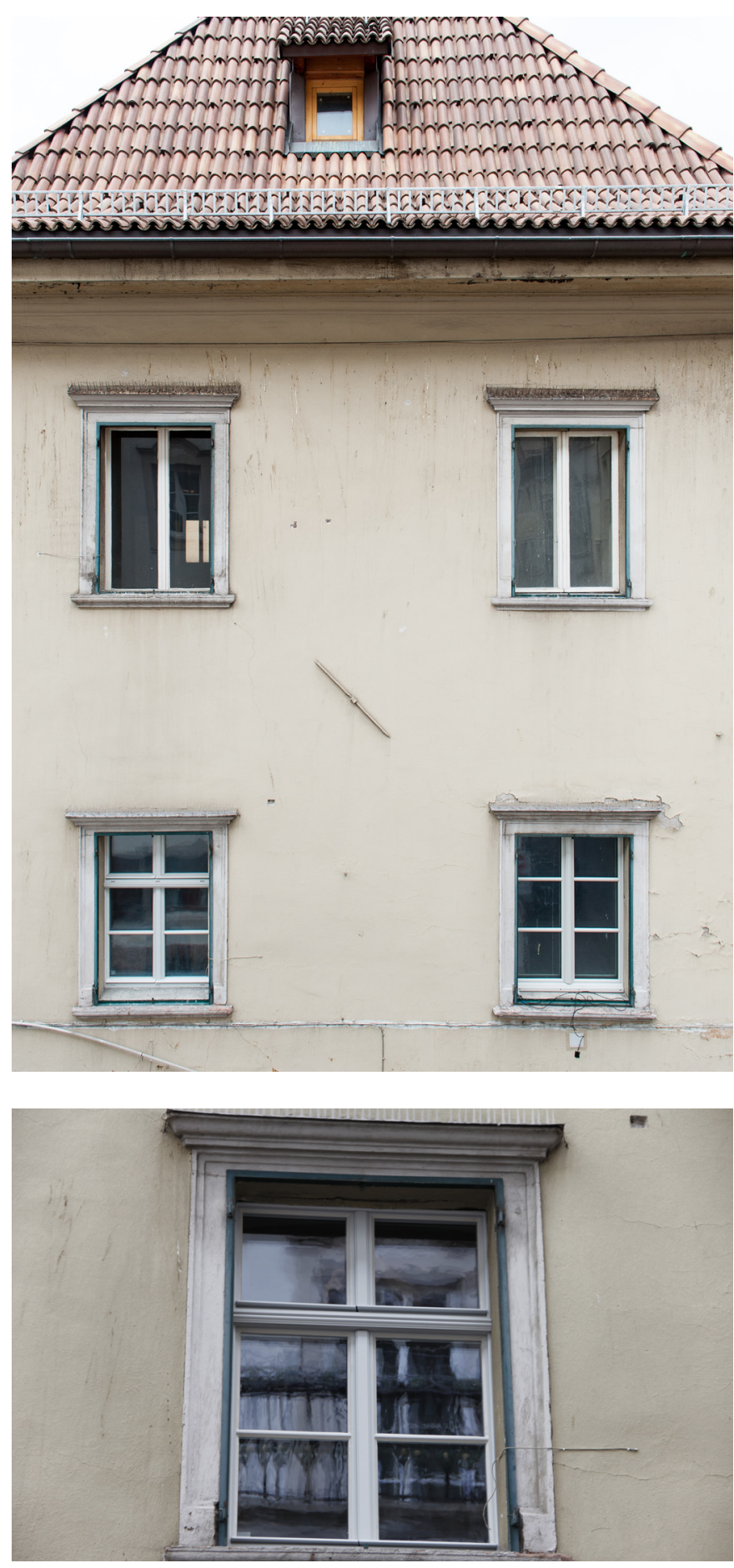

Ventana con eficiencia energética compatible con los edificios históricos | fotos Florian Berger/EURAC 
a debate La eficiencia energética y la edificación histórica | coordinan Mónica López Sánchez, Ana Yáñez Vega

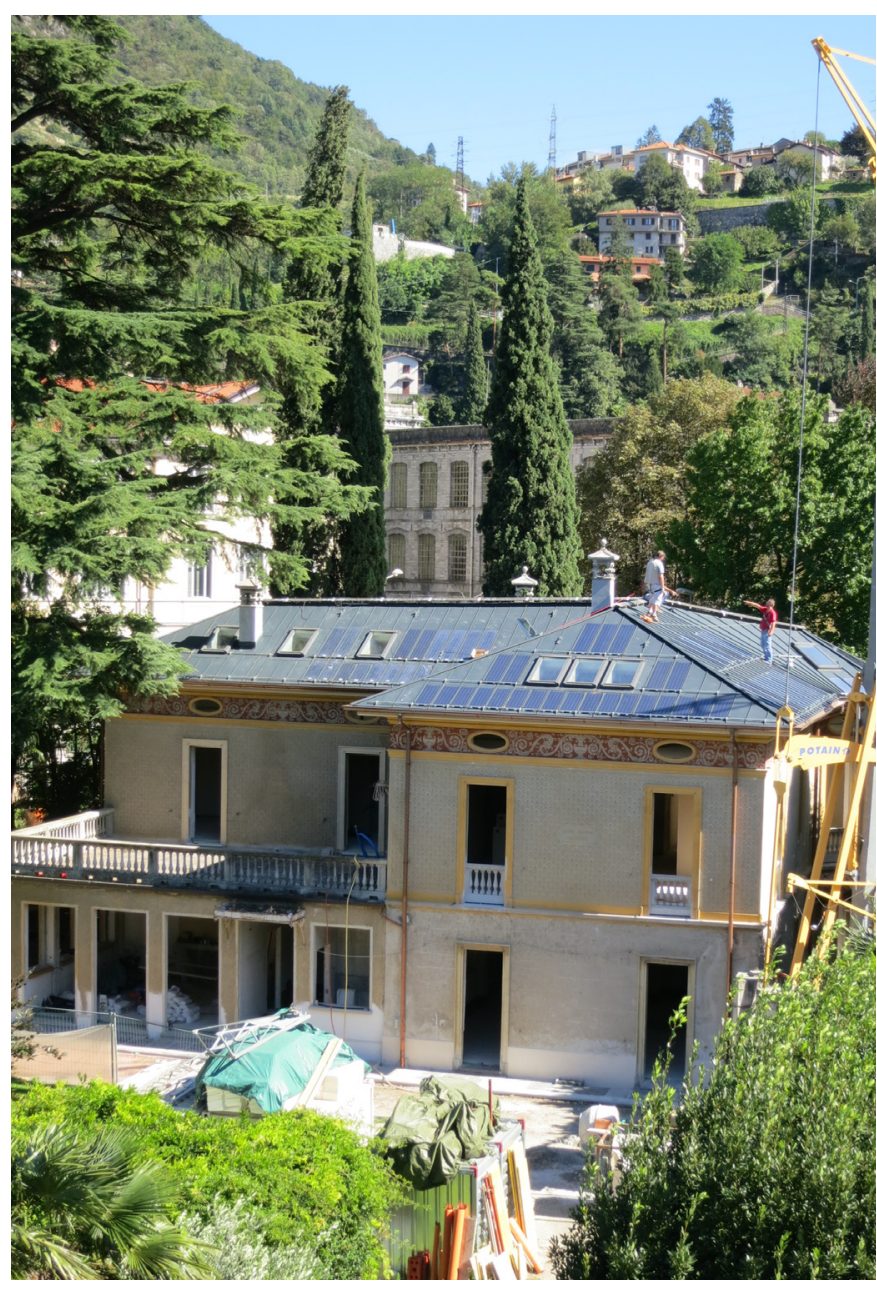

Integración del fotovoltaico en la arquitectura histórica | foto Valentina Carì

res y precisión de instalación (Sustainable Renovation of Historical Buildings - SuRHiB).

\section{Ventanas}

El potencial de mejora energética de una ventana existente es alto. En primer lugar, generalmente su duración es más corta que la del edificio: a menudo los edificios tienen ventanas originales de los siglos XVIII y XIX, pero rara vez del siglo XVII o anteriores. Por eso, es posible implementar las intervenciones de sustitución de puertas y ventanas ya degradados o reemplazados con el tiempo y la creación de nuevos marcos compatibles con el existente. Además de las intervenciones radicales, es posible graduar la acción sustituyendo únicamente el cristal, aplicando películas de baja emisividad, adicionando una doble ventana, colocando cortinas pesadas o oscuras, reparando juntas o sellos. Los experimentos en Inglaterra (ENERGY, 2009; CONSERVATION, 2000) compararon los beneficios relacionados con las tecnologías con un rendimiento diferente (cortinas de tela pesada, persianas, cortinas, film a baja emisividad, mejor sellado, doble ventana). El sistema tradicional más eficaz consiste en una combinación de cristal oscuro y doble (>70\%), seguido de persianas exteriores (>60\%), venecianas y escudos interiores reflectantes (50-60\%) y cortinas pesadas (40-50\%). No es aconsejable, sin embargo, la sustitución de las ventanas tradicionales con marcos en materiales contemporáneos (aluminio o PVC) que modifican la imagen estética de la fachada, y además generan emisiones ambientales durante el ciclo de producción más altos que los beneficios de energía.

\section{Gestión}

Por último, es necesario intervenir con controles y mantenimiento continuos que permitan evaluar la respuesta del edificio, conocer nuevos métodos de intervención y corregir los problemas que se plantean.

\section{CONCLUSIONES}

En conclusión, la mejora de la eficiencia energética de un edificio histórico debe equilibrar el aumento de rendimiento (en términos de consumo de combustible, confort y seguridad) y la conservación, con el objetivo de optimizar las características y el potencial energético y ambiental original. El método sugiere un enfoque estratégico y repetible para conservar y mejorar la eficiencia energética del patrimonio cultural, reconociendo que el proyecto no se limita al diseño del edificio sino que requiere el mantenimiento y la actualización en el tiempo. 
a debate La eficiencia energética y la edificación histórica | coordinan Mónica López Sánchez, Ana Yáñez Vega

\section{BIBLIOGRAFÍA}

- CEI EN 16247-1. Diagnosi energetiche - Parte 1: Requisiti generali, 2012

- The CONSERVATION and Renewal of Timber Windows (2000) Essex: Essex Country Council Planning Department, 2000

- DALL'O', G. (ed.) (2011) Green Energy Audit. Manuale operativo per la diagnosi energetica e ambientale degli edifici. Milano: Edizioni Ambiente, 2011

- EFFICIENZA energetica negli edifici storici (2013) Milano: AICARR (Associazione Italiana Condizionamento dell'Aria, Riscaldamento, Refrigerazione), 2013

- ENERGY conservation in traditional buildings (2009) London: English Heritage, 2009

- HOGELING, J. (2006) The set of CEN standards developed to support the implementation of the EPBD in the EU Member States. En EPBD Buildings Platform, Information Paper P02, April 11, 2006

- IS0 6781 Thermal insulation. Qualitative Detection of Thermal Irregularities in Building Envelopes. Infrared Method (1983) Brussels: European Committee for Standardization, 1983

- ISO 9869 Thermal Insulation. Building Elements. InSitu Measurement of Thermal Resistance and Thermal Transmittance (1994) Brussels: European Committee for Standardization, 1994

- ISO 9972 Thermal performance of buildings. Determination of Air Permeability of Buildings. Fan Pressurization Method (2006) Brussels: European Committee for Standardization, 2006

- LUCCHI, E. (2012) Diagnosi energetica strumentale degli edifici. Palermo: Dario Flaccovio Editore, 2012

- LUCCHI, E.; PRACCHI, V. (ed.) (2013) Efficienza energetica e patrimonio costruito. La sfida del miglioramento delle prestazioni nell'edilizia storica. Santarcangelo di Romagna: Maggioli Editore, 2013

- NYPAN, T. M.; RONCHI, A. M. (ed.) (2006) European legislation and Cultural Heritage. Milano: Delewa, 2006

- PROTOCOLlO di certificazione volontaria GBC Historic BuildingTM (2014) Rovereto: Green Building Council (GBC) Italia, 2014

- La QUALITÀ delle prestazioni energetico-ambientali nella manutenzione dell'architettura storica. Linee Guida (2011) Venezia: MVBC (Metadistretto Veneto dei Beni Culturali), MVB (Metadistretto Veneto della Bioedilizia), 2011)
- SPIEKMAN, M.; VAN DIJK, D. (2011) Relation historic buildings, EPBD and EPBD CEN Standards. Bruxelles: Commissione Europea, 2011

- THUMANN, A.; JOUNGER, W. J. (2008) Handbook of Energy Audits. Lilburn: The Fairmont Press, VII Ed., 2008

\section{LEGISLACIÓN}

- DIRETTIVA 2002/91/CE del Parlamento Europeo e del Consiglio del 16 dicembre 2002 sul rendimento energetico nell'edilizia (2003) Gazzetta ufficiale delle Comunità europee de 4 de enero de 2003, OJ L 1, pp. 65-71

- DIRETTIVA 2010/31/UE del Parlamento Europeo e del Consiglio del 19 maggio 2010 sulla prestazione energetica nell'edilizia (rifusione) (2010) Gazzetta ufficiale delle Comunità europee de 18 de junio de 2010, OJ L 153, pp. 13-35

- DIRETTIVA 2012/27/UE del Parlamento Europeo e del Consiglio del 25 ottobre 2012 sull'efficienza energetica, che modifica le direttive 2009/125/CE e 2010/30/UE e abroga le direttive 2004/8/CE e 2006/32/CE (2012) Gazzetta ufficiale delle Comunità europee de 14 de noviembre de 2012, OJ L 15, pp. $1-56$

- DIRETTIVA 2009/28/CE del Parlamento Europeo e del Consiglio del 23 aprile 2009 sulla promozione dell'uso dell'energia da fonti rinnovabili, recante modifica e successiva abrogazione delle direttive 2001/77/CE e 2003/30/CE (2009) Gazzetta ufficiale delle Comunità europee de 5 de junio de 2009, OJ L 140, pp. 16-62 